\title{
Background History, Clinical Presentation and Laboratory Profile in Cases of Suspected Neurometabolic Disorders
}

\author{
MUSTAFAMAHBUB ${ }^{1}$, AZM MOSIUL AZAM $^{2}$, SURAJ C. MAZUMDER ${ }^{3}$, BITHI DEBNATH $^{4}$, \\ NAILA ZAMAN KHAN 5 .
}

\begin{abstract}
Background : Neurometabolic disorders (NMD) in children may present at any age with a wide range of clinical manifestations. Unexplained or intractable seizure is one of the important associations. Consanguinity, regression of development and sibling death are the clues to suspect neurometabolic disorders when laboratory support is limited. Laboratory findings however, provide the confirmatory diagnosis which is unavailable in Bangladesh.
\end{abstract}

Objectives : To determine the association of consanguinity, regression of development, seizures, EEG findings and other laboratory investigations in children suspected to have neurometabolic disorders and to aid clinicians working in resourcepoor countries.

Methodology : A retrospective analysis was done from the records of the patients suspected to have neurometabolic disorders admitted in the department of Neurosciences, Dhaka Shishu Hospital, Dhaka during the period of July 2007 to February 2011. Tandem Mass Spectrometry (TMS), biotinidase activity and other enzyme assay were done through a private laboratory in New Delhi, India.

Results : Total 128 children were studied and the parents of 39 (31\%) had history of consanguineous marriage. Seizure was associated with 96 (75\%) children and abnormal EEG findings were recorded in 83 (65\%). Plasma ammonia was done in 98 cases and found to be increased in 53 (54\%) cases. Plasma lactate was done in 94 cases and found high in 40 (43\%). TMS were done in 111 (85\%) children and abnormality were found in 70 (63\%) cases. Serum biotinidase activity was advised for 41 children as per TMS result and measured in 25 children of which deficient activity was found in 17(68\%); borderline in 4 (16\%) and normal activity in 4 (16\%) cases.

Conclusion: Background history and clinical presentation followed by stepwise laboratory investigation is necessary to identify neurometabolic disorders. Early and appropriate intervention can reduce neurodisability in many situations.

Key words: Neurometabolic disorders, Tandem Mass Spectrometry, Consanguinity.

1. Associate Professor, Department of Neurosciences, Bangladesh institute of child health $(\mathrm{BICH})$, Dhaka Shishu Hospital, Dhaka.

2. Assistant Professor, Department of Neurosciences, Bangladesh institute of child health $(\mathrm{BICH})$, Dhaka Shishu Hospital, Dhaka.

3. Registrar, Department of Neurosciences, Dhaka Shishu Hospital, Dhaka.

4. FCPS student (child neurology \& development), Department of Neurosciences, Dhaka Shishu Hospital, Dhaka.

5. Professor, Department of Neurosciences, Bangladesh institute of child health (BICH), Dhaka Shishu Hospital, Dhaka.

Correspondence: Dr. Mustafa Mahbub, Associate Professor,

Email: mustafamahbub@yahoo.com

\section{Introduction:}

Inborn errors of metabolism cause hereditary metabolic diseases (HMD) and classically they result from the lack of activity of one or more specific enzymes or defects in the transportation of proteins. The consequences can usually be the accumulation of substances present in small amounts, the deficiency of critical intermediary products, the deficiency of specific final products or furthermore the noxious excess of products of alternative metabolic pathways. 1,2 
Over one third of the inherited metabolic disorders are characterized by the central nervous system involvement and neurological symptoms are the presenting and the most prominent clinical problems associated with them. ${ }^{3}$

The main subgroups of neurological importance are disorders of amino acid and organic acid intermediary metabolism, lysosomal diseases, peroxisomal disorders and errors in energy metabolism especially the mitochondrial diseases. ${ }^{4}$

All IEM are single-gene disorders, almost all being inherited in an autosomal recessive or X-linked recessive manner. ${ }^{5}$ Primary mitochondrial respiratory chain disease is a heterogeneous group of disorders characterized by impaired energy metabolism due to presumed genetically-based oxidative phosphorylation (OXPHOS) dysfunction.

Although primary mitochondrial disease by definition has a genetic etiology, the genetic abnormality may be found in either mitochondrial DNA (mtDNA) or nuclear DNA (nDNA). ${ }^{6}$

Clinical oiinters towards an underlying IEM include: deterioration after a period of apparent normal development, parental consanguinity, family history of unexplained neonatal deaths, rapidly progressive encephalopathy, seizures of unexplained cause, severe metabolic acidosis, persistent vomiting and peculiar odor. ${ }^{7}$

The basic laboratory studies that should be obtained for an infant who has symptoms of a metabolic encephalopathy consistent with an inborn error of metabolism include: Complete blood count with differential, urinalysis, blood gases, serum electrolytes, blood glucose, plasma ammonia, urine reducing substances, urine ketones if acidosis or hypoglycemia present, plasma and urine amino acids, quantitative urine organic acids, plasma lactate. ${ }^{8}$

Tandem mass spectrometry (MS/MS) is a powerful technology that allows a rapid and sensitive screening over more than thirty inborn errors of metabolism through measurement of amino acids and acylcarnitines in a small quantity of blood. ${ }^{9}$

The aim of this study is to determine the association of consanguinity, regression or delay in development, seizures, EEG findings and other/step-wise laboratory investigations (ammonia, lactate and Tandem Mass Spectrometry (TMS)) in children suspected to have neurometabolic disorders, to aid clinicians working in resource-poor countries.

\section{Methodology:}

Retrospectively analysis was done regarding the demographic, clinical symptoms and laboratory (biochemical and electrophysiological) findings of 128 patients admitted in the Department of Pediatric Neurosciences, Dhaka Shishu Hospital during the period from December 2007 to February 2011 suspected of having inborn error of metabolism.

Early suspicion made by the presence of consanguinity, regression of developmental skills, unexplained developmental delay, sibling death, affected sibling and clinical manifestations of unexplained seizure, vomiting, encephalopathy, mental retardation, ataxia, spasticity and cutaneous abnormalities.

Almost all children of suspected neurometabolic disorder underwent a primary screening of serum ammonia, lactate, fasting blood sugar, electrolytes, SGPT, arterial blood gas and urinary ketones. Other tests included complete blood count, neuroimaging (USG, CT, MRI), electroencephalography (EEG). Children who had clinical findings for specific diseases didn't go for ammonia, lactate or TMS estimation eg. cherry red spot for Tay-Sachs disease, KayserFlescher ring for Wilson's disease, demyelination found in neuroimaging for leukodystrophy. Tandem mass spectrometry (TMS) was done where abnormality found in primary screening test. Biotinidase deficiency was suspected on the basis of TMS abnormality in a significant number of cases and serum biotinidase activity measured. Further tests suggested according to abnormalities found in TMS.

Patient's data were evaluated and categorized as age, sex, consanguinity, perinatal insult, sibling death, affected sibling, regression of development, developmental delay, seizure and level of

ammonia, lactate, TMS and biotinidase activity in serum. The data were analyzed using descriptive method and no statistical testing was applied.

\section{Results:}

Among 128 analyzed children, 70 were given presumptive diagnosis of IEM based on positive screening tests. The age and sex wise distribution shows preponderance of males (64\%) compared to females (36\%). Age distribution showed that maximum participants were from the age group $>1$ to 5 years (53\%) and only $16 \%$ cases were from the $>5$ years of age (Table-I). 
BANGLADESH J CHILD HEALTH 2015; VOL 39 (1) : 26

Table-I

Age \& sex wise distribution of participants

\begin{tabular}{llc}
\hline & Total number of cases & 128 \\
\hline Sex & Males & $82(64 \%)$ \\
& Females & $46(36 \%)$ \\
Age & 1 month to 1 year & $39(31 \%)$ \\
& 1 year to 5 years & $68(53 \%)$ \\
& $>$ 5 years & $21(16 \%)$ \\
\hline
\end{tabular}

Available family history and various clinical presentation suggests that the common presentations were seizures(75\%), delayed milestones (51\%), regression of development (41\%), Consanguinity (31\%), sibling deaths (17\%), affected siblings (5\%) and history of perinatal insults (51\%). [Table-II].

Table-II

Back groud history and clinical findings ( $n=128)$

\begin{tabular}{lccc}
\hline & Present & Absent & Not known \\
\hline H/O Consanguinity & $39(31 \%)$ & $88(68 \%)$ & 01 \\
H/O Perinatal insultl & $65(51 \%)$ & $60(47 \%)$ & $03(2 \%)$ \\
Sibling death & $22(17 \%)$ & $83(65 \%)$ & $23(18 \%)$ \\
Affected sibling & $06(5 \%)$ & $98(76 \%)$ & $24(19 \%)$ \\
Regression of & $52(41 \%)$ & $73(57 \%)$ & $03(2 \%)$ \\
development & & & \\
Early developmental & $65(51 \%)$ & $63(49 \%)$ & $03(2 \%)$ \\
delay & & & \\
Seizure & $96(75 \%)$ & $31(24 \%)$ & 01 \\
\hline
\end{tabular}

Seizures were associated with $96(75 \%)$ children and abnormal EEG findings recorded in $83(65 \%)$ [Table -III].

Table-III

EEG findings of the patients $(n=128)$

\begin{tabular}{lc}
\hline EEG findings & Number of Patients \\
\hline Normal & $35(27 \%)$ \\
Localized epileptiform discharge & $30(23 \%)$ \\
Epileptic encephalopathy & $18(14 \%)$ \\
Diffuse encephalopathy & $15(12 \%)$ \\
Generelized epileptiform discharge & $11(8.4 \%)$ \\
Generelized encephalopathy & $6(4.6 \%)$ \\
Severe encephalopathy & $1(0.8 \%)$ \\
Localized encephalopathy & $1(0.8 \%)$ \\
Multifocal epileptiform discharge & $1(0.8 \%)$ \\
Not found & $10(7.8 \%)$ \\
\hline Total & $128(100 \%)$ \\
\hline
\end{tabular}

Background History, Clinical Presentation and Laboratory Profile

Common laboratory findings were increased serum Ammonia in (42\%) and increased serum Lactate in (31\%) Tandem mass Spectrometry (TMS) was done in 111 patients where 70 (55\%) cases showed abnormal findings suggestive of some neurometabolic disorder. (Table - IV).

Table-IV

Serum Ammonia, Lactate level and TMS abnormality $(n=128)$

\begin{tabular}{lccc}
\hline & Ammonia & Lactate & TMS \\
\hline Done & $98(77 \%)$ & $94(73 \%)$ & $111(87 \%)$ \\
Increased /abnormal & $53(54 \%)$ & $40(43 \%)$ & $70(63 \%)$ \\
Normal & $45(46 \%)$ & $54(57 \%)$ & $41(37 \%)$ \\
\hline
\end{tabular}

The suspected neurometabolic disorders based on the TMS abnormality shows deficient biotinidase activity in 41 cases.[Table-V].

Table-V

Suspected neurometabolic disorders on the basis of TMS abnormality $(n=70)$

\begin{tabular}{lc}
\hline Disorder & Number \\
\hline Biotinidase deficiency & $41(59 \%)$ \\
Urea cycle disorder & $9(12 \%)$ \\
Low carnitine & $9(12 \%)$ \\
Medium chain acyl-CoA dehydrogenase & $5(7 \%)$ \\
deficiency (MCADD) & $2(3 \%)$ \\
HHH syndrome & $2(3 \%)$ \\
Multiple CoA Carboxylase deficiency & $1(1.5 \%)$ \\
Mitochondrial disorder & $1(1.5 \%)$ \\
Homocystinuria & 70 \\
\hline Total & \\
\hline
\end{tabular}

Serum biotinidase activity was done in 28 cases, deficient activity found in 17, borderline activity in 4 and normal activity found in 4 cases. [Table - VI].

Table-VI

Estimation of Biotinidase activity and result $(n=41)$

\begin{tabular}{lc}
\hline Suggested & 41 \\
Done & $28(68 \%)$ \\
Deficient activity & $17(60 \%)$ \\
Borderline activity & $4(14 \%)$ \\
Within normal range & $4(14 \%)$ \\
Not found & $3(11 \%)$ \\
\hline
\end{tabular}




\section{Discussion:}

The investigation of infants and children with suspected inborn errors of metabolism and specifically inherited neurometabolic disorders is challenging in pediatric neurology practice.

Each infant/child deserves a detailed clinical and individual assessment. There is no standard battery of laboratory tests; thus, experience and multidisciplinary teamwork are of the utmost importance. ${ }^{10}$ The male predominance is un-explained except for a possible gender bias in hospitalization of sick children.

Background history, mode of presentation and clinical examination was the important part of the initial evaluation. In the history there may be several important factors to suggest an IEM. The previous family history should be explored for evidence of consanguinity and unexplained neonatal or infantile deaths; this should include a three generation pedigree. ${ }^{11}$ Most inborn errors of metabolism are inherited in an autosomal recessive fashion. In most of these cases, the disease can only manifest itself fully in homozygotes for the mutation. This is generally a rare occurrence, except in consanguineous families ${ }^{12} \mathrm{~A}$ positive family history and consanguinity were the most important pointers. In this study 39 (31\%) cases had parental consanguinity. In Egypt, inborn errors of metabolism are common, presumably because of the high rates of consanguinity (38\%). ${ }^{13}$

Regression of development is one of the most important clinical presentation for the suspicion of NMD. The common scenario associated with metabolic disease is that of the child or infant who initially develops and grows well before developing symptoms. In our study 52 (41\%) cases were presented with regression of previously acquired developmental skills. Developmental regression is significant and always needs investigation. ${ }^{14}$

The commoner causes of developmental delay are chromosomal abnormalities and structural brain abnormalities ${ }^{15}$ One of the challenging questions for paediatricians investigating children with developmental delay is how intensively should one investigate for inborn errors of metabolism and which metabolic investigations are indicated.

Reports on the prevalence of inborn errors of metabolism (IEMs) as the underlying cause of delay show some variation but generally indicate that IEMs are found to be the cause in only a small percentage of these cases (that is, $1-5 \%$ ). ${ }^{16}$

Seizures were a predominant symptom among our study subjects. Seizures were associated with 96 (75\%) children and abnormal EEG findings recorded in $83(65 \%)$. Seizures are more likely to be uncontrolled in metabolic disorder.

Study from Vidya et al in India shows seizure in 26\% cases. ${ }^{17}$ Higher values (43\%) were reported by Ilhem and coworkers, in Tunisia. ${ }^{18}$

There is lack of metabolic screening Facilities in developing countries. A battery of four simple tests namely arterial blood gases, blood ammonia, urinary ketones and blood lactate are advocated in tertiary health care centers ${ }^{19}$ Elevation of blood ammonia is usually caused by defects of urea cycle enzymes. Infants with elevated blood ammonia levels from urea cycle defects commonly have normal serum $\mathrm{pH}$ and bicarbonate values. Elevation of ammonia is also observed in some infants with certain organic acidemias Elevated lactate along with ketoacidosis with or without hyperammonnemiais seen in lactic acidosis. Ketoacidosis with or without elevated lactate along with hyperammonnemia points towards organic aciduria. ${ }^{19,20}$

The inborn errors of metabolism detected by tandem mass spectrometry generally include aminoacidemias, urea cycle disorders, organic acidurias, and fatty acid oxidation disorders. Earlier recognition of these inborn errors of metabolism has the potential to reduce morbidity and mortality rates in these infants. $^{21}$

Among 128 cases of suspected neurometabolic disorder TMS was done in 111 cases and abnormality found in 70 (63\%).Considering on the nature of abnormal values, biotinidase deficiency was suggested for 41 (59\%) and urea cycle disorder for 9 (13\%) cases. Study from Fayza, et al in Egypt shows among 550 OP cases presenting to the neurometabolic clinic, 24 cases with IEM were detected $(1 / 23 ; 4.3 \%) 19$ cases with aminoacid disorders (1/28) and 5 cases with organic acidemias (1/110). ${ }^{22}$

Slightly higher values were reported by Xie and coworkers, in China (11\%). ${ }^{23}$ In Omani population, Joshi et al. detected $32 \%$ IEM by TMS in the metabolic unit. The authors reported that parental

Biotin is a very important vitamin that found in some foods. ${ }^{25}$ Biotinidase deficiency is a rare and treatable 
inherited neurometabolic disorder with an estimated incidence of 1:61, 067 population. ${ }^{26}$ This disorder in its severe form is much rarer with incidence of $1: 137401 .^{27}$ In this study most of the cases were suspected as biotinidase deficiency based on TMS abnormality.

A precise diagnosis of neurometabolic disorder requires specific test. In our study specific enzyme estimation is done only in limited number of patients due to both unavailability of the test in our country or unaffordibility of the parents. Among the suspected disorders only biotinidase activity was done.

\section{Conclusion:}

Background history and clinical presentation followed by stepwise laboratory investigation is necessary to identify neurometabolic disorders. Definitive diagnosis by specified tests can be advised for those patients who are positive on screening tests. Early and appropriate intervention can reduce neurodisability in many situations consanguinity was twice as frequent in the study patients as compared to the general population.

\section{References:}

1. Sanderson S, Green A, Preece MA, Burton H. The incidence of inherited metabolic disorders in the West Midlands, UK. Arch Dis Child 2006; 91: 896-99.

2. Martins AM.Inborn errors of metabolism: a clinical overview. Sao Paulo Med J 1999; 117:251)

3. Rita C, Bindu PS. An insight into the biochemistry of inborn error of metabolism for a clinical neurologist: Ann Indian Acad Neurol 2008; 11 : 68-81.

4. Aicardi J, Ogier H. Metabolic Diseases. In: Aicardi J, Diseases of the Nervous System in Childhood, $3^{\text {rd }}$ ed. London, Mc Keith Press. 2009. p 243-326.

5. Jewell CW. Inborn Errors of Metabolism of Acute Onset in Infancy. Pediatrics in Review 1990. 11. P. 205-216.

6. Haas RH, Parikh S, Falk M J, Russell P. S, Wolf NI. Darin N et al. Mitochondrial Disease: A Practical Approach for Primary Care Physicians. Pediatrics 2007. 120: 1326-1333.

7. Suvasini S, Pradeep K, Ramesh A, Madhulika $K$, Ashok D, Vinod Paul. Approach to Inborn
Errors of Metabolism Presenting in the Neonate; AlIMS- NICU protocols 2010.

8. Barbara K B. Inborn Errors of Metabolism in Infancy: A Guide to diagnosis: paediatrics. 1998: 102. p. 69.

9. Wenjun $\mathrm{T}$, Jian $\mathrm{H}$, Fang $\mathrm{D}$, Xinyu $\mathrm{W}$, Ying $\mathrm{L}$. Impact of Inborn Errors of Metabolism on Admission in a Neonatal Intensive Care Unit- $A$ Prospective Cohort Study. Indian J Pediatr ; 2012;79:494-500.

10. Goknur H, Emine V, Leyla B. When do we need to perform a diagnostic lumbar puncture for neurometabolic diseases/ Positive yield and retrospective analysis from a tertiary center. The Turkish journal of Pediatrics 2012; 54: 52-58.

11. Cleary MA, Green A, Developmental delay: when to suspect and how to investigate for an inborn error of metabolism. Arch Dis Child 2005; 90:1128-32.

12. Decker BC. Inborn Errors of Metabolism In: Bernard L. Maria. Current Management in Child Neurology. Third Edition 2005, P. 311-316.

13. Temtamy SA, Kandil MR, Demerdash AM, Hassan WA, Meguid NA, Afifi $\mathrm{HH}$, An epidemiological/ genetic study of mental subnormality in Assiut Governorate. Egypt. Clin Genet. 1994; 46: 347- 51.

14. Germaine P.Neurodegenerative disorders and metabolic disease, Arch Dis Child 2013;98:8.

15. Majnemer A, Shevell MI. Diagnostic yield of the neurologic assessment of the developmentally delayed child. J Pediatr 1995;127:193-9.

16. Curry CJ, Stevenson RE, Aughton D. Evaluation of mental retardation: recommendations of a consensus conference: American College of Medical Genetics. Am J Med Genet 1997; 72:468-77.

17. Patil VS., Jailkhani R, Trivedi DJ, Kulkarni SP, Sagare AA, Mudaraddi $R$ et al. Screening for aminoacidurias and organic acidurias in patients with metabolic or neurological manifestations. Biomedical Research 2012; 23: 253-258.

18. Youssef IB, Kraoua I, Smirani S, Mariem K, Ben RH, Rouissi A et al. Epilepsy Aspects and EEG Patterns in Neuro-Metabolic Diseases. Journal of Behavioral and Brain Science 2011; 1: 69-74. 
19. Sheffali G, Meena V, Veena K, Madhulika K, Manjeet K: An approach to Neurometabolic Disorders by a simple Metabolic Screen. Indian Pediatrics 2000;37 : 63-69.

20 Razvani I, Razvani G. An approach to Inborn errors of Metabolism. In ; Behrman RE, Kliegman RM, Jenson HB, Editors. Nelson Textbook of Pediatrics.19 th $^{\text {th }}$ ed. Philadelphia,WB Saunders; 2011: p.416-18.

21. Talkad S. Inborn Errors of Metabolism in Infancy and Early Childhood: An Update. Am Fam Physician 2006;73:1981-90.

22. Fayza $H$, Fatma EM, Iman $M$, Sawsan $H$, Laila S, Sahar AA. Preliminary Results of Egypt Experience for Use of Tandem Mass Spectrometry for Expanded Metabolic Screening. Journal of Applied Sciences Research 2009; 5: 1425-35.
23. Xie LJ, Zhu JX, Zhu XD, Li HJ, Han LS, Gu XF. Combined use of tandem mass spectrometry with urine gas chromatography/mass spectrometry is useful for diagnosis of inborn errors of metabolism in children. Zhongguo Dang Dai Er Ke Za Zhi 2008 ;10:31-4.

24. Joshi SN, Hashim J, Venugopalan P. 2002. Pattern of inborn errors of metabolism in an Omani population of the Arabian Peninsula. Annals of Tropical Pediatrics 2002; 22: 93- 96.

25. Karimzadeh P, Ahmadabadi F, Jafari N, Jabbehdari S, Alaee MR, Ghofrani M. Biotinidase Deficiency: A Reversible Neurometabolic Disorder. Iran J Child Neurol. 2013; 7:47- 52.

26. Rathi N, Rathi M. Biotinidase deficiency with hypertonia as unusual feature. Indian Pediatr 2009;46:65-67.

27. Wolf $B$. Worldwide survey of neonatal screening for biotinidase deficiency. J Inherit Metab Dis 1991;14:923-7. 